\title{
Nanoencapsulation of bovine lactoferrin for food and biopharmaceutical applications
}

\author{
Victor M. Balcão a,b,d,*, Carla I. Costa a , Carla M. Matos ${ }^{\mathrm{a}}$, Carla G. Moutinho ${ }^{\mathrm{a}, \mathrm{b}}$, Manuela Amorim ${ }^{\mathrm{c}}$, \\ Manuela E. Pintado ${ }^{c}$, Ana P. Gomes ${ }^{c}$, Marta M. Vila ${ }^{d}$, José A. Teixeira ${ }^{b}$ \\ ${ }^{a}$ Universidade Fernando Pessoa, Rua Carlos da Maia $n^{\circ}$ 296, P-4200-150 Porto, Portugal \\ ${ }^{\mathrm{b}}$ Instituto para a Biotecnologia e a Bioengenharia - IBB, Centro de Engenharia Biológica, Universidade do Minho, Campus de Gualtar, P-4710-057 Braga, Portugal \\ ${ }^{\mathrm{c}}$ Escola Superior de Biotecnologia, Universidade Católica Portuguesa, Rua Dr. António Bernardino de Almeida, P-4200-072 Porto, Portugal \\ ${ }^{\mathrm{d}}$ Laboratório para o Desenvolvimento e Avaliação de Substâncias Bioactivas, Universidade de Sorocaba, Cidade Universitária, Rod. Raposo Tavares km 92.5, \\ 18023-000 Sorocaba, São Paulo, Brazil
}

\section{A R T I C L E I N F O}

\section{Article history:}

Received 20 July 2012

Accepted 7 February 2013

\section{Keywords:}

Lactoferrin

Lipid nanovesicles

Multiple water-in-oil-in-water emulsions

Structural stabilization

Antimicrobial activity

\begin{abstract}
A B S T R A C T
Lactoferrin has for long captured the interest of many researchers as a natural compound with a wide variety of uses. Lactoferrin is a monomeric, iron-binding $80 \mathrm{kDa}$ glycoprotein, and appears to be the subfraction of whey with the best documented antiviral, antimicrobial, anticancer and immune modulating/enhancing effects. It belongs to the family of transferrin proteins, and serves to control iron levels in body fluids by sequestering and solubilizing ferric iron. In the present research effort, production of lactoferrin derivatives (starting from a purified commercial extract), encompassing full stabilization of its three-dimensional structure, has been attempted via nanoencapsulation within lipid nanovesicles, integrating a multiple water-in-oil-in-water emulsion. Long-term storage of the multiple nanoemulsions produced did not lead to leaching of protein, thus proving the effectiveness of the encapsulation procedure. Furthermore, lactoferrin nanovesicle derivatives prepared under optimal conditions were successfully employed at lab-scale antimicrobial trials.
\end{abstract}

(C) 2013 Elsevier Ltd. All rights reserved.

\section{Introduction}

Lactoferrin is an iron transporter, glycoprotein, isolated from bovine milk, constituting a minor component of whey proteins. This protein is composed of 703 aminoacids and has a molecular weight of ca. 80 kDa (Hoek, Milne, Grieve, Dionysius, \& Smith, 1997). Lactoferrin is considered a multifunctional protein, playing several biological roles. Due to its iron-binding properties, lactoferrin potentially plays a role in iron uptake by the intestinal mucosa of the suckling neonate, hence making it a potential source of iron for breast-fed infants. On the other hand, lactoferrin has been shown to have antibacterial, antiviral, antifungal, anti-inflammatory, antioxidant and immunomodulatory activities (Hoek et al., 1997; Orsi, 2004; Rodríguez-Franco, Vásquez-Moreno, \& Montfort, 2005; Takakura et al., 2003; Ward, Uribe-Luna, \& Conneely, 2002; Yamauchi, Tomita, Giehl, \& Ellison, 1993). Lactoferrin can show a

\footnotetext{
* Corresponding author. Laboratory for the Development and Evaluation of Bioactive Substances, University of Sorocaba, Cidade Universitária, Rod. Raposo Tavares km 92.5, CEP 18023-000 Sorocaba, São Paulo, Brazil. Tel.: +55 1199478 5858; fax: +551521017000.

E-mail addresses: vbalcao@ufp.edu.pt, vbalcao@gmail.com (V.M. Balcão).
}

direct bactericidal activity due to its binding to the lipid A part of bacterial lipopolyssacharide, with an associated increase in membrane permeability, an action that is due to lactoferricin, a peptide obtained from lactoferrin by enzymatic cleavage, which is active not only against bacteria, but even against fungi, protozoa and viruses (Orsi, 2004). Bovine lactoferrin, extracted from whey proteins, is marketed as a nutritional supplement, but has a high potential for biopharmaceutical applications. However, as most proteins or enzymes, it is fragile, and even small conformational changes may reduce its activity, and therefore stabilisation is required. One possibility is to encapsulate the protein into nanometre-sized vesicles to protect the bioactive protein from denaturation by proteolysis and dilution effects. The development of strategies that may permit structural and functional stabilization of lactoferrin via nanoencapsulation may increase the biopharmaceutical applicability of this bioactive protein (Srinivas et al., 2010). Such lactoferrinencasing nanovesicles could be utilized, e.g. in the formulation of dental elixirs to eliminate the oral microflora responsible for formation of oral biofilms with one clear advantage: the adhesive nanovesicles could slowly liberate its naturally antimicrobial content, thus reducing the development of dental cavities. Oral delivery of proteins has been long identified as one of the main challenges in 
drug delivery science and technology (Almeida \& Souto, 2007; García-Fuentes, Torres, \& Alonso, 2002; Lee \& Yuk, 2007; Reis, Neufeld, Ribeiro, \& Veiga, 2006a; Sanjula, Shah, Javed, \& Alka, 2009), since in addition to its great technological potential, important drawbacks for its development still exist (Yang, Yuan, \& Jin, 2009). Currently, the number of drugs based on large biological molecules, such as proteins and peptides, is drastically increasing (Reis, Neufeld, Ribeiro, \& Veiga, 2006b). However, various factors, including stability and circulation time in the gastrointestinal tract, can strongly affect the effective absorption of oral-delivered proteins (Dupeyrón, Rieumont, González, \& Castaño, 2009).

Lipid-based carriers have attracted increasing scientific and commercial attention during the last few years as alternative materials for the delivery of peptides and proteins concerned with stability issues (Acosta, 2009; Chakraborty, Shukla, Mishra, \& Singh, 2009; Rawat, Singh, Saraf, \& Saraf, 2008). Recently, a newly developed technique for stabilization of proteins and enzymes based on nanoencapsulation procedures has started to gain momentum, based on encapsulation of macromolecules in a nanoporous matrix by the gelation of a solution (Erdinc, 2007, pp. 90; Ferrer, Yuste, Rojo, \& del Monte, 2003; Ragoonanan \& Aksan, 2007). Due to being entrapped in a very confined environment (i.e. in the nanopores), the molecular motions of water molecules are altered, slowing down. Therefore, confinement of proteins and/or peptides within nanoporous matrices (or coatings) has the same effect as the osmolytes with respect to changing the water activity and therefore modifying the biochemical reaction rates. Due to increased viscosity and entropic confinement (physical entrapment), the (degradation) reaction rates are slowed down and the biological materials can be stabilized for storage (Mozafari et al., 2008; Ragoonanan \& Aksan, 2007). Water-in-oil-in-water (W/O/W) emulsions are examples of multiple emulsions, in which dispersions of small water droplets within larger oil droplets are themselves dispersed in a continuous aqueous phase (Bibette, Calderon, \& Poulin, 1999; Dupeyrón et al., 2009; Ficheux, Bonakdar, LealCalderon, \& Bibette, 1998; García-Fuentes et al., 2002; Hanson et al., 2008; Wang, Tao, \& Gang, 2006). Emulsions are quite utilized in many ways of processing, and are extensively used by the food, cosmetic, and coating industries. Due to their compartmentalized internal structure, multiple emulsions present advantages over simple oil-in-water $(\mathrm{O} / \mathrm{W})$ emulsions for encapsulation, such as the ability to carry both polar and non-polar molecules, and a better control over releasing of therapeutic molecules (Davis \& Walker, 1987; Marcato \& Durán, 2008; Okochi \& Nakano, 2000; Pays, Giermanska-Kahn, Pouligny, Bibette, \& Leal-Calderon, 2002). In the present research work, the potential of natural antimicrobial, bovine lactoferrin, stabilized via nanoencapsulation for incorporation in a mouth wash elixir has been investigated. Bovine lactoferrin was entrapped within $\mathrm{W} / \mathrm{O} / \mathrm{W}$ multiple nanoemulsions, aiming at mimicking the multifunctional design of biological membranes, with several lipid matrices, and stabilizing layer compositions. The composition of the stabilizing layer of the nanosystem was changed by using different proportions of lecithin and different poloxamers (nonionic triblock copolymers with a central hydrophobic core of polyoxypropylene flanked by two hydrophilic chains of polyoxyethylene), thus allowing to control degradation times of the nanovesicles. For the production of nanoemulsions, bioactive compounds were previously dissolved in the aqueous phase prior to be dispersed in the melted lipid during homogenization in an UltraTurrax (model T25D from IKA) under heating (ca. $40{ }^{\circ} \mathrm{C}$ ). The resulting nanoemulsions were subsequently fully characterized physicochemically. Characterization of the optimized nanovesicle formulations encasing the bioactive lactoferrin encompassed determination of particle size, size distribution and particle charge, via Zeta potential analysis, surface morphology via Scanning Electron Microscopy (SEM), and thermal analysis via Differential Scanning Calorimetry (DSC). The antimicrobial activity of the nanoemulsions was also assessed in vitro, upon several microbial strains in liquid medium, including pathogenic bacteria and Candida albicans.

\section{Materials and methods}

\subsection{Materials}

\subsubsection{Bioactive protein}

The bioactive lactoferrin used consisted of a purified extract (protein content of $96.0 \%$, of which lactoferrin accounts for $98.0 \%$ ) from bovine whey, and was kindly supplied by DMV International (Delhi NY, USA).

\subsubsection{Chemicals}

Softisan $100^{\mathrm{TM}}$ was purchased from Sasol (Sasol Olefins \& Surfactants GmbH, Hamburg, Germany). Precirol ATO5 (glycerol distearate) and Compritol 888ATO (glycerol dibehenate) were kindly supplied from Gattefossé SAS (Saint Priest, France). Glycerol (anhydrous) was purchased from Fluka (Steinheim, Germany). Surfactants: Tween 80 was purchased from Sigma-Aldrich (St. Louis MO, USA). Lutrol F68 ${ }^{\mathrm{TM}}$ (poloxamer 188) and Lutrol F127 were kindly supplied by BASF ChemTrade GmbH (Ludwigshafen, Germany). Other chemicals: $\mathrm{CaCl}_{2} \cdot 2 \mathrm{H}_{2} \mathrm{O}$ was obtained from Riedelde-Haën (Seelze, Germany. Commercial $\mathrm{HCl}(37 \%)$ and $\mathrm{NaCl}$ were purchased from Vaz Pereira (Lisbon, Portugal). Soybean phosphatidylcholine (lecithin) was purchased from Acofarma (Barcelona, Spain). Filter paper $(0.22 \mu \mathrm{m})$ was purchased from Nalgene (New York NY, USA), and nonsterile filters $(0.45 \mu \mathrm{m})$ were purchased from Nucleopore (Cambridge MA, USA). Tap water was purified in a Milli-Q Plus 185 system (Molsheim, France) to a final conductivity of ca. $18.2 \mathrm{M} \Omega \mathrm{cm}^{-1}$. The solvents used were all analytical grade or better, and were used without further purification.

\subsubsection{Analytical equipment}

All spectrophotometric readings were carried out using quartz cuvettes in an UV-VIS spectrophotometer from Shimadzu (Kyoto, Japan). The Zeta Potential and Hydrodynamic Size of the nanoemulsion particles were determined in a Zetasizer (model Nanoseries Nano-ZS) from Malvern Instruments Ltd (Worcestershire, United Kingdom). Calorimetric studies were carried out in a differential scanning calorimeter (Shimadzu, Kyoto, Japan), which comprised a detector (DSC-50) and a thermal analyser (TA-501). Cryo-SEM analysis of lipid nanoemulsions were carried out in a Scanning Electron Microscope from JEOL (model JSM 6301F from JEOL, Tokyo, Japan), coupled with an Ion Sputter-Coater JSC1100 (JEOL, Tokyo, Japan), an INCA 350 Energy X-Ray microanalysis system via Energy Dispersive Spectrometry (SEM/EDS) from Oxford Instrument $\mathrm{GmbH}$ (Wiesbaden, Germany), and a cryo transfer system from Gatan Inc. (model Alto 2500 from Gatan Inc, Pleasanton CA, USA).

\subsection{Experimental procedures}

\subsubsection{Preparation of multiple lactoferrin-encasing nanoemulsions}

Production of multiple nanoemulsions (water-in-oil-in-water, $\mathrm{W} / \mathrm{O} / \mathrm{W}$ ) with nanoencapsulated lactoferrin were carried out in duplicate in an UltraTurrax (model T25D from IKA) under heating (ca. $40^{\circ} \mathrm{C}$ ), with the bioactive lactoferrin being previously dissolved in the (inner) aqueous phase (W) prior to being dispersed in the melted lipid (O) (Softisan) during homogenization (10 min at $8000 \mathrm{rpm}$ ). Therefore, final $\mathrm{W} / \mathrm{O} / \mathrm{W}$ dispersions of lactoferrin were obtained via sequential (optimized) homogenization of a $\mathrm{W} / \mathrm{O}$ 
dispersion involving two cycles for $10 \mathrm{~min}$. The inner aqueous phase was constituted by $\mathrm{HCl} 10 \mathrm{mM}, \mathrm{CaCl}_{2} 20 \mathrm{mM}$, Tween 80 and pure bovine lactoferrin (with concentrations ranging from $2.5 \mathrm{mg} /$ $\mathrm{mL}$ to $25 \mathrm{mg} / \mathrm{mL}$ ); the intermediate oily phase encompassed glycerol, Softisan (or Precirol or Compritol) and soybean phosphatidylcholine; finally, the outer aqueous phase encompassed Lutrol F68 (or Lutrol F127), ultrapure water and $\mathrm{NaCl} 10 \mathrm{mM}$. Control nanoparticles were also produced, without lactoferrin, and stored for 52 days under the same conditions. The solid lipids were tested as possible constituents of the oily phase. All these agents are considered to be lipids for modified release formulations. The formulations prepared with each of these lipids showed a milky and uniform appearance.

\subsubsection{Evaluation of the combined effects of homogenization time,} lactoferrin concentration and ionic strength of the outer aqueous phase, for optimization of the nanoformulation

Stability of the multiple nanoemulsions was evaluated by producing emulsions as result of the combined effect of homogenization time (5, 10 and $20 \mathrm{~min})$, lactoferrin concentration (2.5, 5.0, 10.0 and $25.0 \mathrm{mg} / \mathrm{mL})$ and ionic strength $(\mathrm{NaCl} \mathrm{1,10} \mathrm{and} 100 \mathrm{mM})$ of the aqueous outer phase.

\subsubsection{Determination of hydrodynamic size and Zeta potential}

Zeta potential is the electric potential that prevails in the cutting plan of a particle, which is at a small distance from its surface, and which results from the measure of the mobility distribution of a dispersion of charged particles as they are subject to an electric field. The mobility can be defined as the velocity of a particle per unit electric field, and is determined by applying an electric field to the dispersion of particles and measuring their average velocity. The results obtained (in duplicate) allow determination of the hydrodynamic size of the lipid nanovesicles, of the polydispersion index and of their Zeta potential.

\subsubsection{Thermal analysis by differential scanning calorimetry (DSC)}

For every calorimetric assay, ca. $10 \mathrm{mg}$ of (nano)emulsion were weighed (using a microsyringe) directly into the interior of highpressure aluminium pans (Shimadzu, P/N 557 201-53090, 64U3670609), and duly sealed by pressure. A reference aluminium pan was also prepared by simply sealing air inside an empty case. The samples were then heated from room temperature to $100{ }^{\circ} \mathrm{C}$ at a constant linear scanning rate of $5{ }^{\circ} \mathrm{C} \mathrm{min}-1$, during which the amount of heat absorbed by the samples was recorded.

\subsubsection{Antimicrobial activity of lactoferrin and multiple nanoemulsions}

Screening of antimicrobial activity was determined by the diffusion method. Inocula of each target microorganism - Staphylococcus aureus, Salmonella sp., Escherichia coli, Pseudomonas aeruginosa, Listeria innocua, Bacillus cereus, and C. albicans - were prepared by suspension of a pure colony in $0.9 \%$ sterile $\mathrm{NaCl}$ (to reach a standard turbidimetry of 0.5 McFarland). Each suspension was inoculated in Mueller Hinton Agar, and after drying, separated wells were made in the plates, and $60 \mu \mathrm{L}$ of different nanoemulsion samples and controls were added. Sterile water was used as negative control, and antibiotics as positive controls (chloramphenicol for S. aureus, Salmonella sp., E. coli, P. aeruginosa, and $B$. cereus and ampicillin for L. innocua). Each plate was incubated at $37^{\circ} \mathrm{C}$ for $24 \mathrm{~h}$, after which the inhibition halos were measured. In the case of pure lactoferrin and nanoemulsion samples with antimicrobial activity, Minimum Inhibitory Concentration (MIC) was determined by microplate assay, using same target microorganisms and inocula as described previously. Each suspension of microorganism was inoculated in 96-well plates containing Mueller Hinton
Broth for bacteria and Yeast Malt Broth for yeast, with different lactoferrin concentrations (2000, 1000, 500, 250, 200, 125, $62.5 \mu \mathrm{g} \mathrm{mL}^{-1}$ ). Sterile water was used as negative control and uninoculated media were used as a positive control. The plates were incubated at $37{ }^{\circ} \mathrm{C}$ for $24 \mathrm{~h}$, and optical density was measured by plate reader at $620 \mathrm{~nm}$. The MIC was determined as the lowest concentration of lactoferrin (free or in nanoemulsion) that showed absence of turbidimetry when compared with the positive control.

\subsection{6. (Cryo-) scanning electron microscopy analyses}

Lipid nanoemulsions encasing lactoferrin were analysed for microstructural and morphological characteristics via Cryo-SEM. Briefly, lactoferrin-encasing nanoemulsion samples were prepared for analysis as follows: (i) samples were mounted in an appropriate (aluminium) support inserted into a gold-coated plate; (ii) the gold-coated plate was then duly fixed in a transfer stick; (iii) the support containing the sample was immersed in liquid (slush) nitrogen; (iv) the sample was then transferred under vacuum into the SEM preparation chamber (also under vacuum and maintained cold via addition of liquid nitrogen); (v) inside the chamber, the deep-frozen sample was fractured, undergone sublimation (during $90 \mathrm{~s}-300 \mathrm{~s}$ ) by gently increasing the temperature from $-140{ }^{\circ} \mathrm{C}$ to ca. $-90{ }^{\circ} \mathrm{C}$, and was sputter-coated with $100-200 \AA$ of $\mathrm{Au} / \mathrm{Pd}$ (during $20 \mathrm{~s}$ ) in a strong vacuum $(0.5 \mathrm{~atm})$ between 20 and $30 \mathrm{~mA}$ at $2.5 \mathrm{kV}$; (vi) finally, the sample was transferred into the SEM chamber for microscopy analysis. The samples were analysed at CEMUP (Centro de Materiais da Universidade do Porto) with a scanning electron microscope from JEOL operating at 5-15 kV, with an incidence angle of $30^{\circ}$, coupled with X-Ray microanalysis. A cryo transfer system for SEM analysis was used for all sample transfer prior to SEM analysis.

\section{Results and discussion}

\section{1. (Cryo-) scanning electron microscopy}

Magnifications of pure lactoferrin powder of $400 \times$ and of lactoferrin-encasing nanoemulsions of $15,000 \times$ and $50,000 \times$ are depicted in Fig. 1a-c, respectively. The cryo scanning electron microphotographs of the exposed (fractured) surface of lactoferrinencasing nanoemulsions (see Fig. 1b and c) show the poloxamer matrix structure developed during the manufacturing process and the highly homogeneous distribution of nanovesicle diameters.

\subsection{Optimization of the formulation}

For producing an optimal nanoformulation, several variables were studied, viz. lipid nature, poloxamer concentration, soy lecithin concentration, homogenization time, and lactoferrin concentration (see Table 1). (Purified) buffered lactoferrin was found to be more stable than unbuffered lactoferrin. Centrifugation of the optimized nanoformulations did not lead to any lactoferrin liberation induced by bursting nanovesicles (as revealed by UV-VIS spectrophotometry), thus evidencing the firm encapsulation and concomitant structural stabilization of lactoferrin. Increasing lactoferrin concentration led to an increase in the values of Zeta potential (from negative towards positive values). Addition of an electrolyte (sodium chloride) to the external aqueous phase also led to an increase in the values of Zeta potential (see Fig. 2), presumably due to accumulation of adsorbed ions at the particle surfaces. However, when using a higher $(100 \mathrm{mM})$ electrolyte concentration, phase separation was notorious immediately after homogenization. Addition of electrolyte at concentrations of $10 \mathrm{mM}$ proved to be most suitable, by producing nanovesicles with (more) stable Zeta potential values over time. 

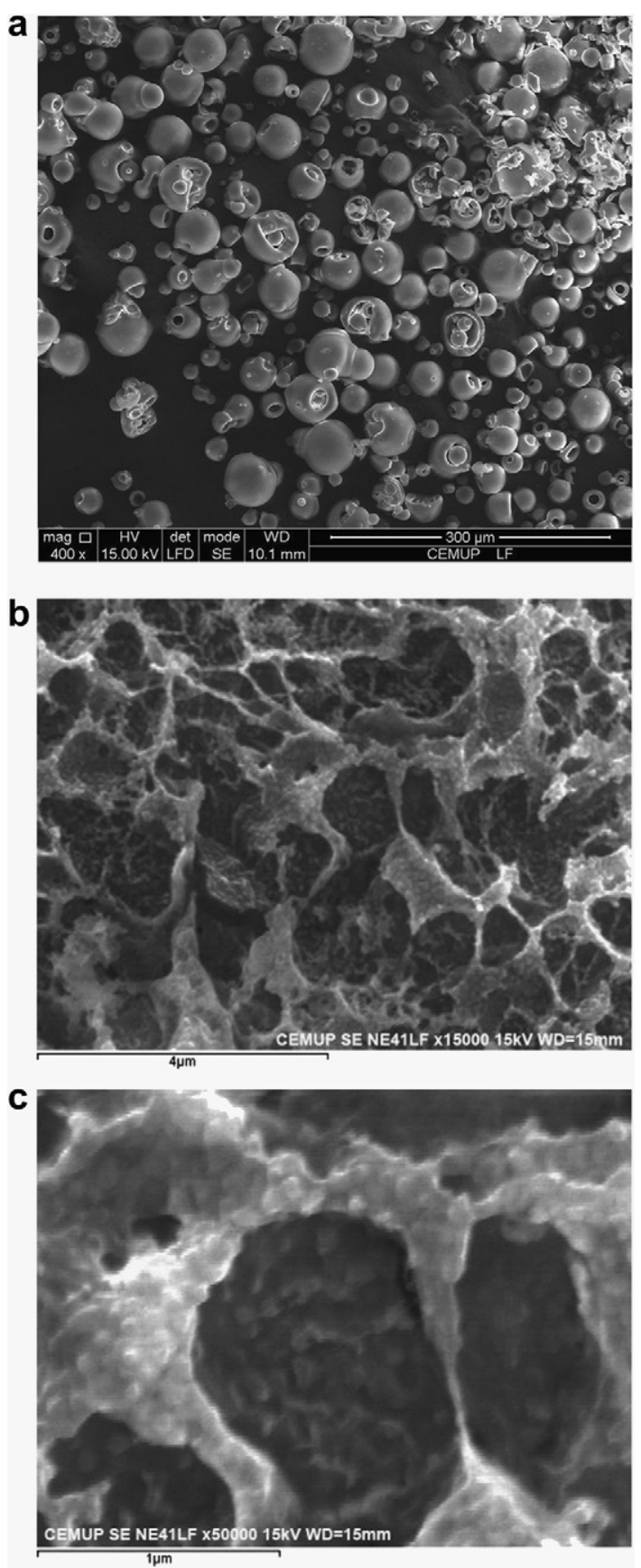

Fig. 1. Scanning electron microscopy analysis of the lactoferrin lyophilizate using $\times 400$ magnification (a) and cryo-scanning electron microphotographs of lactoferrinencasing nanoemulsions, showing the poloxamer network in the outer aqueous phase after sublimation and sputter coating with gold/palladium, for $\times 15,000$ magnification (b) and $\times 50,000$ magnification (c).

\subsection{Hydrodynamic size and Zeta Potential}

The net charge at the surface of particles in suspension affects the ionic distribution in their immediate surroundings, producing an electrical double layer around each particle. When a particle moves, the ions within the boundary move with it, and vice versa. Zeta potential is the potential that exists at this boundary, and its intensity is an indication of the potential stability in the colloidal system, and can be measured by determination of the linear velocity of the particles in an electric field. The Zeta potential depends on the concentration of ions in the solvent. The main reason why to measure the Zeta potential lies in predicting colloidal stability, which in turn depends on the interactions between particles. Zeta potential is therefore an attempt to quantify such interactions, by predicting stability. The Zeta potential is a measure of the repulsive forces between particles, and since the majority of colloidal aqueous systems is stabilized via electrostatic repulsion, the larger the repulsive forces between particles the smaller the probability for them to become closer and form aggregates, leading to a more stable colloidal system. The mean size and size distribution (polydispersity index) were measured in duplicate samples via Dynamic Laser light Scattering (suitable for particles ranging from $5 \mathrm{~nm}$ to ca. $3 \mu \mathrm{m}$ ), and the results presented as the mean values. Optimum homogenization time was found to be $10 \mathrm{~min}$, at $8000 \mathrm{rpm}$, since during such homogenization timeframe the particle size was maintained at values ranging from 100 to $200 \mathrm{~nm}$ (see Figs. 2 and 3). Storage of the optimized multiple nanoemulsions (produced with Softisan) throughout 50 days at room temperature led to an increase in the values of Zeta potential, presumably due to ion concentration at particle interface (see Fig. 2). Control nanoparticles were also produced, without lactoferrin, and stored for 52 days under the same conditions; for these, a slight increase in particle size was noticed, together with a slight increase in Zeta potential values. Regarding particle size, no change was observed in the range of $100-200 \mathrm{~nm}$. Precirol ${ }^{\mathrm{TM}}$ and Compritol ${ }^{\mathrm{TM}}$ were also tested as lipids for the oily phase, but owing to their high(er) melting points, were discarded in order to prevent deactivation of lactoferrin (see Fig. 2). Lecithin and Tween 80 were selected as suitable excipients, since even at duplicate amounts the Zeta potential values were maintained at lower (negative) values at the same time that hydrodynamic size did not suffer any significative changes over time (see Fig. 2). Increasing electrolyte concentration leads to an increase in the values of Zeta potential, presumably due to accumulation of adsorbed ions at the particle surfaces. Addition of electrolyte at concentrations of $10 \mathrm{mM}$ proved to be most suitable by producing nanovesicles with stable Zeta potential over time. Storage of the optimized multiple nanoemulsions (with Softisan, due to its mild melting temperature) throughout several days at room temperature led to an increase in Zeta potential values (see Fig. 4), presumably due to ion concentration at particle interface.

\subsection{Antimicrobial activity}

Probably, the best known role of lactoferrin is as an iron-binding protein, as it is known to have an extremely high affinity for this metal. Many pathogenic bacteria need a supply of free iron to multiply and in the presence of lactoferrin are strongly inhibited or killed. The antimicrobial activity of the different nanoemulsions, assessed in vitro against several microbial strains showed different efficiency depending on the pathogenic microorganism. The antimicrobial activity of both free and encapsulated lactoferrin was validated through in vitro procedures against several microbial strains, including pathogenic bacteria and yeast $C$. albicans. The results showed that the MIC of both free and encapsulated lactoferrin upon the bacteria $S$. aureus and L. inoccua was $2000 \mu \mathrm{g} \mathrm{mL}^{-1}$ while that for $B$. cereus was higher than $2000 \mu \mathrm{g} \mathrm{mL}^{-1}$. In the case of yeast $C$. albicans, it was susceptible to lower concentrations of lactoferrin, since the MIC was $200 \mu \mathrm{g} \mathrm{mL}^{-1}$. The results were similar to both free and encapsulated lactoferrin, in the same concentration basis. It has been demonstrated (Ellison, Giehl, \& LaForce, 1988) that lactoferrin interacts with Gram negative bacteria leading to the release of significant levels of lipopolysaccharide from the outer membrane, compromising the outer membrane permeability and increasing bacterial susceptibility to other antimicrobial molecules such as lysozyme. In the research effort entertained herein, lactoferrin did not exhibit any antimicrobial activity upon Gram negative 
Table 1

Optimization of processing conditions leading to an optimal nanoformulation.

\begin{tabular}{|c|c|c|c|c|c|c|c|c|}
\hline \multicolumn{2}{|c|}{ Formulation parameters } & \multicolumn{7}{|c|}{ Composition of the several nanoformulations (w/w) } \\
\hline & & \multirow{3}{*}{$\begin{array}{l}\begin{array}{l}\text { Designed starting } \\
\text { conditions }\end{array} \\
8000 \\
0.05 \%\end{array}$} & \multirow{3}{*}{$\begin{array}{l}\text { Without lactoferrin } \\
\text { (control) } \\
8000 \\
0.00 \%\end{array}$} & \multirow{3}{*}{$\begin{array}{l}\text { With lactoferrin } \\
\text { and } \mathrm{NaCl} \\
8000 \\
0.05 \%\end{array}$} & \multirow{3}{*}{$\begin{array}{l}\text { With lactoferrin, } \\
\mathrm{NaCl} \text { and } \mathrm{CaCl}_{2} \\
8000 \\
0.05 \%\end{array}$} & \multirow{3}{*}{$\begin{array}{l}\text { With } 25 \% \text { more of } \\
\text { lecithin and tween } 80 \\
8000 \\
0.05 \%\end{array}$} & \multicolumn{2}{|c|}{$\begin{array}{l}\text { With } 50 \% \text { more lecithin } \\
\text { and Tween } 80\end{array}$} \\
\hline \multicolumn{2}{|c|}{ Homogenization speed (rpm) } & & & & & & 8000 & Best formulation \\
\hline Internal aqueous & Lactoferrin & & & & & & $0.05 \%$ & achieved \\
\hline \multirow[t]{3}{*}{ phase } & $\mathrm{HCl}(0.010 \mathrm{M})$ & $9.80 \%$ & $9.80 \%$ & $9.79 \%$ & $9.79 \%$ & $9.79 \%$ & $9.78 \%$ & \\
\hline & $\mathrm{CaCl}_{2}(0.020 \mathrm{M})$ & $0.00 \%$ & $0.00 \%$ & $0.00 \%$ & $0.03 \%$ & $0.03 \%$ & $0.03 \%$ & \\
\hline & Tween 80 & $0.10 \%$ & $0.10 \%$ & $0.10 \%$ & $0.10 \%$ & $0.12 \%$ & $0.15 \%$ & \\
\hline \multirow[t]{3}{*}{ Oil phase } & Lipid & $0.98 \%$ & $0.98 \%$ & $0.98 \%$ & $0.98 \%$ & $0.98 \%$ & $0.98 \%$ & \\
\hline & Soybean lecithin & $0.10 \%$ & $0.10 \%$ & $0.10 \%$ & $0.10 \%$ & $0.12 \%$ & $0.15 \%$ & \\
\hline & Glycerol & $9.80 \%$ & $9.80 \%$ & $9.79 \%$ & $9.79 \%$ & $9.79 \%$ & $9.78 \%$ & \\
\hline \multirow[t]{3}{*}{$\begin{array}{l}\text { External aqueous } \\
\text { phase }\end{array}$} & $\begin{array}{l}\text { Poloxamer } \\
\text { (Lutrol F68) }\end{array}$ & $0.78 \%$ & $0.78 \%$ & $0.78 \%$ & $0.78 \%$ & $0.78 \%$ & $0.78 \%$ & \\
\hline & Ultrapure $\mathrm{H}_{2} \mathrm{O}$ & $78.39 \%$ & $78.44 \%$ & $78.36 \%$ & $78.32 \%$ & $78.29 \%$ & $78.25 \%$ & \\
\hline & $\mathrm{NaCl}(0.010 \mathrm{M})$ & $0.00 \%$ & $0.00 \%$ & $0.05 \%$ & $0.05 \%$ & $0.05 \%$ & $0.05 \%$ & \\
\hline
\end{tabular}

bacteria (Salmonella sp, E. coli, Pseudomonas fluorescens) since they were not inhibited, but as mentioned above it may have destabilized their outer membrane.

\subsection{Microcalorimetric analysis}

Differential scanning calorimetric measurements provided an insight into the state and degree of crystallinity, and the melting and crystallization behaviour of crystalline materials such as the lipid nanovesicles produced. The amount of heat absorbed by the lipid nanovesicles was the same when comparing empty vesicles and vesicles incorporating lactoferrin, for an (optimized) homogenization period of $10 \mathrm{~min}$ (see Fig. 5). Also, increasing homogenization time led to nanovesicles that exhibited an increase in the amount of heat absorbed by the particles, probably due to a reduction in size and/or to liberation of lactoferrin (see Figs. 3 and 5 ). The effect of homogenization time was very notorious in the amount of heat absorbed (melting enthalpy) by the lipid nanoformulation encasing lactoferrin: $75.48 \mathrm{~J} \mathrm{~g}_{\text {nanoemulsion }}^{-1}$ for $5 \mathrm{~min}$ homogenization time (absorption peak at $91.70{ }^{\circ} \mathrm{C}$ ), $245.04 \mathrm{~J} \mathrm{~g}_{\text {nanoemulsion }}^{-1}$ for $10 \mathrm{~min}$ homogenization time (absorption peak at $87.64{ }^{\circ} \mathrm{C}$ ), and $866.64 \mathrm{~J} \mathrm{~g}_{\text {nanoemulsion }}^{-1}$ for $20 \mathrm{~min}$ homogenization time (absorption peak at $86.03{ }^{\circ} \mathrm{C}$ ). Increasing

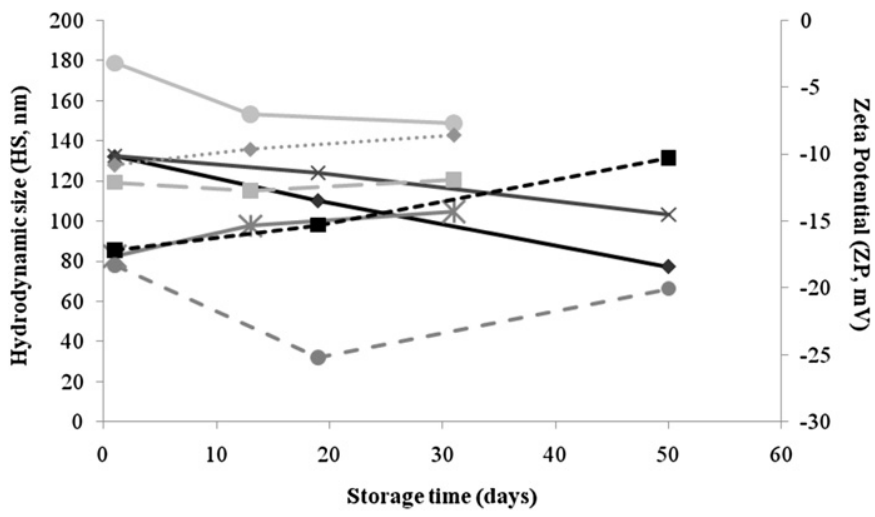

$$
\begin{aligned}
& \text {-Compritol (HS) } \\
& \text {-7-Compritol (ZP) } \\
& \text { - }- \text { Precirol(HS) } \\
& \rightarrow \text { Softisan, with } \mathrm{NaCl} \text { (HS) } \\
& \text { - - Precirol(ZP) } \\
& \cdots * \text { Softisan, without } \mathrm{NaCl} \text { (ZP) } \\
& \text { - Softisan, with } \mathrm{NaCl} \text { (ZP) }
\end{aligned}
$$

Fig. 2. Effect of storage time upon hydrodynamic size and Zeta potential of nanovesicles produced with several (different) lipids, for optimized homogenization timeframe (10 $\mathrm{min})$. homogenization time led to production of nanovesicles that exhibited an increase in the amount of heat absorbed by the particles, probably due to a reduction in size and/or to liberation of lactoferrin (see Fig. 5). A blank (empty) nanoemulsion, prepared without lactoferrin under the same experimental conditions, absorbed $1450 \mathrm{Jg}_{\text {nanoemulsion }}^{-1}$ for $10 \mathrm{~min}$ homogenization time (absorption peak at $87.54^{\circ} \mathrm{C}$ ), a value that is 6 times higher than that of the same nanoemulsion encasing lactoferrin. Inclusion of lactoferrin in the nanoformulation did not lead to significative changes in the peak of heat absorption $\left(87.64^{\circ} \mathrm{C}\right)$, when compared with the blank (empty) counterpart $\left(87.54{ }^{\circ} \mathrm{C}\right)$, but for the same homogenization timeframe the amount of heat absorbed was significatively lower. Lactoferrin alone in ultrapure water showed a melting enthalpy of $878.79 \mathrm{~J} \mathrm{~g}_{\text {nanoemulsion, }}^{-1}$ at a temperature peak of $69.97{ }^{\circ} \mathrm{C}$, a value clearly higher than that of the nanoemulsion encasing lactoferrin after 10 min of homogenization, which probably accounts for the stabilizing effect of the nanoencapsulation procedure. Macroscopic observations of optimized nanosystems with increased amount of the semicrystalline polymer (Tween 80 , i.e. poly(oxyethylene) sorbitan monooleate) showed no visible phase separation, and absence of adherence to the container walls even after a prolonged storage at room temperature. The optimized nanosystem encompassed nanovesicles with an average size of ca. 100-200 nm and an average Zeta Potential of ca. $-14.6 \mathrm{mV}$ (see Figs. 2 and 4), which were maintained stable over a storage

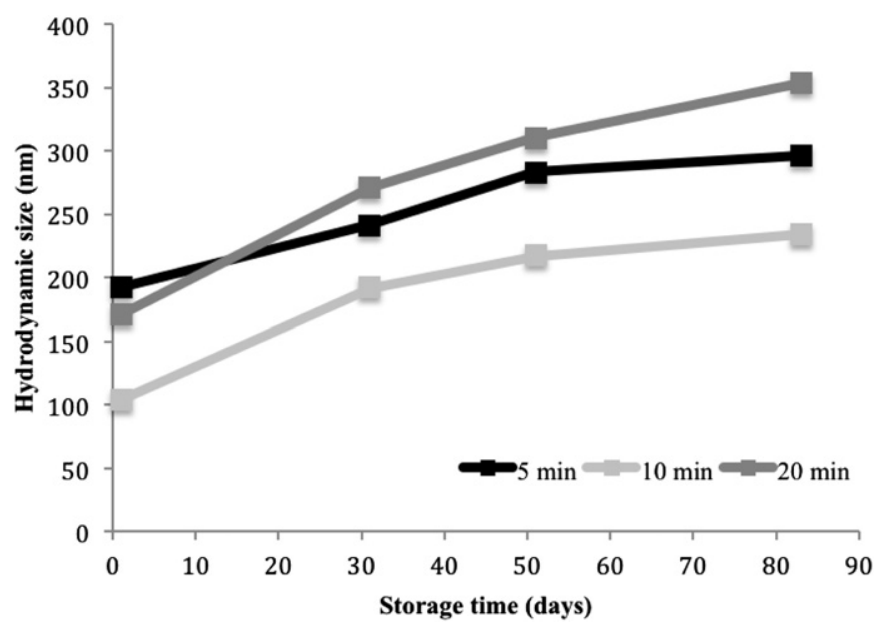

Fig. 3. Effect of homogenization time in the hydrodynamic size of the nanovesicles produced throughout storage time. 


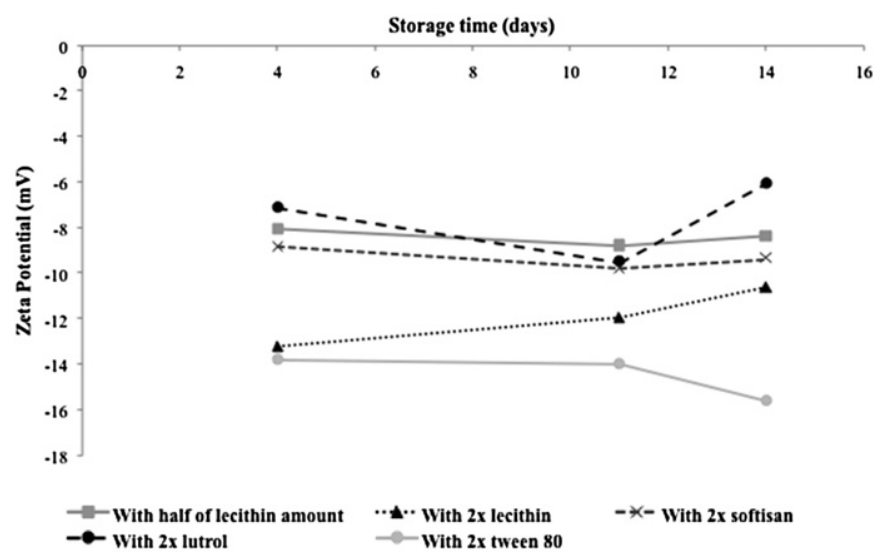

Fig. 4. Effect of different excipients in the Zeta potential of the nanovesicles using Softisan for $10 \mathrm{~min}$ homogenization time.

timeframe of nearly 3 months. Compatibility between increased amounts of Tween 80 and other components of internal aqueous phase can be attributed to hydrogen bonding and to the lower crystalline lattice energy of this polymer, which had a notorious impact in the melting profile of the optimized nanoemulsion. In water-in-oil emulsions (such as our nanovesicles), there is a positive correlation between emulsion stability and fatty acid chain length and a negative correlation with the dielectric constant of the emulsifier. Increasing molecular weight (such as in Softisan $100^{\mathrm{TM}}$, with $\mathrm{C}_{10}-\mathrm{C}_{18}$ fatty acid moieties) and decreasing dielectric constant (such as in Tween 80) indicates greater hydrophobicity, leading to a greater impregnation of the interface and to a more stable (nano) emulsion, which is in clear agreement with the long-term stability observed for our multiple nanoemulsion systems. Additionally, imprisonment of a protein molecule leads to thermodynamic stability, which can be correlated with a change in the thermodynamic conditions of the microenvironment surrounding each molecule, since the movements of (aqueous) solvent molecules in their microneighborhood become seriously reduced by the effect of being contained within the matrix's core. The result is that the protein entity's rotational, translational and vibrational viscosity becomes enhanced, leading to a more rigid three-dimensional architecture with concomitant decrease of entropy and producing stabilization of the biomolecule.

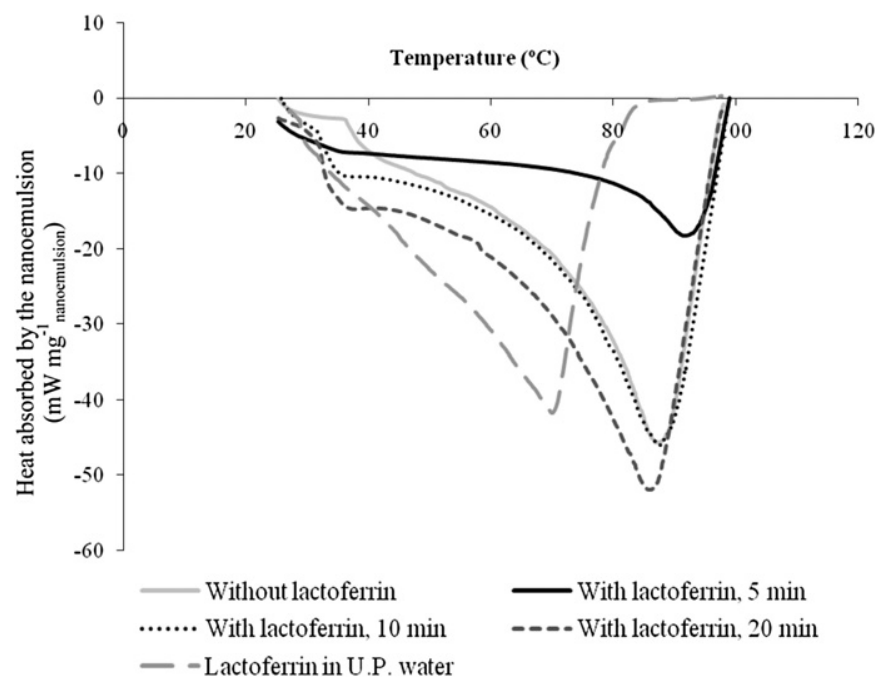

Fig. 5. Differential scanning calorimetry thermograms of multiple nanoemulsions produced during several homogenization times, with and without lactoferrin.

\section{Conclusions}

In this research effort, development and optimization of lipid nanovesicles encasing lactoferrin was pursued. A lipid with mild melting temperature was found most appropriate for the discontinuous oily phase. A homogenisation timeframe of $10 \mathrm{~min}$, the use of an electrolyte with a low ionic strength, and low lactoferrin concentrations were found to be critical variables for producing stable nanovesicle dispersions with diameters ranging from 100 to $200 \mathrm{~nm}$ and Zeta potential values of ca. $-14.6 \mathrm{mV}$. The use of these multiple nanoemulsions in formulating elixirs for oral hygiene would possess inherent advantages, when compared with the current chemical antimicrobial formulation, in that lactoferrin is a naturally harmless protein with bacteriostatic activity, without toxicological risk even for small children.

\section{Acknowledgements}

Financial support from Fundação Ensino e Cultura Fernando Pessoa (FECFP, Porto, Portugal) and from Fundação para a Ciência e a Tecnologia (FCT, Lisbon, Portugal) as pluriannual funding, is gratefully acknowledged. Financial support to Victor M. Balcão, via an Invited Research Scientist fellowship (FAPESP Ref. No. 2011/ 51077-8) by Fundação de Amparo à Pesquisa do Estado de São Paulo (FAPESP, São Paulo, Brazil), is hereby gratefully acknowledged. The authors are also grateful to Dr. Madalena Vieira (affiliated with the IBB) for technical help.

\section{References}

Acosta, E. (2009). Bioavailability of nanoparticles in nutrient and nutraceutical delivery. Current Opinion in Colloid and Interface Science, 14, 3-15.

Almeida, A. J., \& Souto, E. (2007). Solid lipid nanoparticles as a drug delivery system for peptides and proteins. Advanced Drug Delivery Reviews, 59, 478-490.

Bibette, J., Calderon, F. L., \& Poulin, P. (1999). Emulsions: basic principles. Reports on Progress in Physics, 62, 969-1033.

Chakraborty, S., Shukla, D., Mishra, B., \& Singh, S. (2009). Lipid - an emerging platform for oral delivery of drugs with poor bioavailability. European Journal of Pharmaceutics and Biopharmaceutics, 73, 1-15.

Davis, S. S., \& Walker, I. M. (1987). Multiple emulsions as targetable delivery systems. Methods in Enzymology, 149, 51-64.

Dupeyrón, D., Rieumont, J., González, M., \& Castaño, V. M. (2009). Protein delivery by enteric copolymer nanoparticles. Journal of Dispersion Science and Technology, 30, 1188-1194.

Ellison, R. T., III, Giehl, T. J., \& LaForce, F. M. (1988). Damage of the outer membrane of enteric gram-negative bacteria by lactoferrin and transferrin. Infection and Immunity, 56, 2774-2781.

Erdinc, B. I. (2007). Micro/nanoencapsulation of proteins within alginate/chitosan matrix by spray drying. Kingston, Ontario, Canada: Queen's University. M.Sc. Thesis.

Ferrer, M. L., Yuste, L., Rojo, F., \& del Monte, F. (2003). Biocompatible sol-gel route for encapsulation of living bacteria in organically modified silica matrixes. Chemistry of Materials, 15, 3614-3618.

Ficheux, M. F., Bonakdar, L., Leal-Calderon, F., \& Bibette, J. (1998). Some stability criteria for double emulsions. Langmuir, 14, 2702-2706.

García-Fuentes, M., Torres, D., \& Alonso, M. J. (2002). Design of lipid nanoparticles for the oral delivery of hydrophilic macromolecules. Colloids and Surfaces B: Biointerfaces, 27, 159-168.

Hanson, J. A., Chang, C. B., Graves, S. M., Li, Z., Mason, T. G., \& Deming, T. J. (2008). Nanoscale double emulsions stabilized by single-component block copolypeptides. Nature, $455,85-88$.

Hoek, K. S., Milne, J. M., Grieve, P. A., Dionysius, D. A., \& Smith, R. (1997). Antibacterial activity of bovine lactoferrin-derived peptides. Antimicrobial Agents and Chemotherapy, 41, 54-59.

Lee, K. Y., \& Yuk, S. H. (2007). Polymeric protein delivery systems. Progress in Polymer Science, 32, 669-697.

Marcato, P. D., \& Durán, N. (2008). New aspects of nanopharmaceutical delivery systems. Journal of Nanoscience and Nanotechnology, 8, 1-14.

Mozafari, M. R., Khosravi-Darani, K., Borazan, G. G., Cui, J., Pardakhty, A., \& Yurdugul, S. (2008). Encapsulation of food ingredients using nanoliposome technology. International Journal of Food Properties, 11, 833-844.

Okochi, H., \& Nakano, M. (2000). Preparation and evaluation of W/O/W type emulsions containing vancomycin. Advanced Drug Delivery Reviews, 45, 5-26.

Orsi, N. (2004). The antimicrobial activity of lactoferrin: current status and perspectives. BioMetals, 17, 189-196. 
Pays, K., Giermanska-Kahn, J., Pouligny, B., Bibette, J., \& Leal-Calderon, F. (2002). Double emulsions: how does release occur? Journal of Controlled Release, 79, 193-205.

Ragoonanan, V., \& Aksan, A. (2007). Protein stabilization. Transfusion Medicine and Hemotherapy, 34, 246-252.

Rawat, M., Singh, D., Saraf, S., \& Saraf, S. (2008). Lipid carriers: a versatile delivery vehicle for proteins and peptides. Yakugaku Zasshi (The Pharmaceutical Society of Japan), 128, 269-280.

Reis, C. P., Neufeld, R. J., Ribeiro, A. J., \& Veiga, F. (2006a). Nanoencapsulation II. Biomedical applications and current status of peptide and protein nanoparticulate delivery systems. Nanomedicine: Nanotechnology, Biology, and Medicine, 2, 53-65.

Reis, C. P., Neufeld, R. J., Ribeiro, A. J., \& Veiga, F. (2006b). Nanoencapsulation I. Methods for preparation of drug-loaded polymeric nanoparticles. Nanomedicine: Nanotechnology, Biology, and Medicine, 2, 8-21.

Rodríguez-Franco, D. A., Vásquez-Moreno, L. \& Montfort, G. R.-C. (2005). Actividad antimicrobiana de la lactoferrina: mecanismos y aplicaciones clínicas potenciales. Revista Latinoamericana de Microbiologia, 47, 102-111.
Sanjula, B., Shah, F. M., Javed, A., \& Alka, A. (2009). Effect of poloxamer 188 on lymphatic uptake of carvedilol-loaded solid lipid nanoparticles for bioavailability enhancement. Journal of Drug Targeting, 17, 249-256.

Srinivas, P. R., Philbert, M., Vu, T. Q., Huang, Q., Kokini, J. L., Saos, E., et al. (2010). Nanotechnology research: applications in nutritional sciences. Journal of Nutrition, 140, 119-124.

Takakura, N., Wakabayashi, H., Ishibashi, H., Teraguchi, S., Tamura, Y., Yamaguchi, H., et al. (2003). Oral lactoferrin treatment of experimental oral Candidiasis in mice. Antimicrobial Agents and Chemotherapy, 47, 2619-2623.

Wang, Y. F., Tao, Z., \& Gang, H. (2006). Structural evolution of polymer-stabilized double emulsions. Langmuir, 22, 67-73.

Ward, P. P., Uribe-Luna, S., \& Conneely, O. (2002). Lactoferrin and host defense. Biochemistry and Cell Biology, 80, 95-102.

Yamauchi, K., Tomita, M., Giehl, T. J., \& Ellison, R. T. (1993). Antibacterial activity of lactoferrin and a pepsin-derived lactoferrin peptide fragment. Infection and Immunity, 61, 719-728.

Yang, S., Yuan, W., \& Jin, T. (2009). Formulating protein therapeutics into particulate forms. Expert Opinion on Drug Delivery, 6, 1123-1133. 